\title{
Fear and loathing in VR: the emotional and physiological effects of immersive games
}

\author{
Jeroen S. Lemmens ${ }^{1} \mathbb{D} \cdot$ Monika Simon $^{1} \cdot$ Sindy R. Sumter ${ }^{1}$
}

Received: 5 September 2020 / Accepted: 22 June 2021 / Published online: 5 July 2021

(c) The Author(s) 2021

\begin{abstract}
Compared to traditional screen-based media, virtual reality (VR) generally leads to stronger feelings of presence. The current study aimed to investigate whether playing games in VR resulted in a stronger sense of presence than playing on a TV, and whether these feelings of presence affected players' emotional and physiological responses to the games. Two experiments were conducted among 128 students, comparing the effects of playing either a survival horror game $(N=59)$ or a first-person shooter $(N=69)$ on a TV or in VR on physiological and subjective fear, hostility and enjoyment. Results showed that playing games in VR resulted in a stronger sense of presence, lower heart rate variability and a stronger subjective sense of fear. The feeling of presence thereby mediated the effects of VR on fear. The effects of playing a first-person shooter in VR on hostility were mixed, and gaming in VR was not more enjoyable than on TV. Regardless of the type of game or display medium, hostility increased significantly post-play. This study provides evidence that commercial VR games can affect feelings of presence and the physiological and emotional state of players.
\end{abstract}

Keywords Virtual reality $\cdot$ Video games $\cdot$ Presence $\cdot$ Fear $\cdot$ Aggression $\cdot$ Physiological $\cdot$ Measurements

\section{Introduction}

In the past decade, virtual reality (VR) has been successfully applied to treat various psychological problems, including social anxiety (Emmelkamp et al. 2020) fear of flying (Cardo et al. 2017), post-traumatic stress disorder (Botella et al. 2015), and fear of heights (Diemer et al. 2016). The effectiveness of VR as a therapeutic tool, particularly in the context of exposure therapy, is mainly attributed to the immersive quality of this technology. Highly immersive technological systems such as VR lead to a strong sense of situational presence, which can trigger emotional reactions identical to those experienced in similar real-life situations (Alghamdi et al. 2017). Repeated exposure to virtual experiences and subsequent emotional responses reduce negative

Jeroen S. Lemmens

j.s.lemmens@uva.nl

Monika Simon

m.simon@uva.nl

Sindy R. Sumter

s.r.sumter@uva.nl

1 Amsterdam School of Communication Research, University of Amsterdam, Amsterdam, The Netherlands affective symptoms in real life (Cardo et al. 2017). Although studies have shown that VR can facilitate a strong sense of presence and corresponding emotional responses in a therapeutic context, it remains unclear how these insights generalize to VR experiences in an entertainment context. The aim of this study is to examine the emotional impact of commercial VR games.

Despite the relative commercial success of VR gaming systems, research on players' emotional response to headmounted VR systems is scarce. It is expected that playing games in VR will lead to a stronger sense of presence and thereby a more intense emotional experience than regular screen-based gaming, similar to non-game applications of this immersive technology (e.g., Riva et al. 2007). In games, players actively seek out stressful events, frightening enemies, and life-threatening situations, where the thrill of vicariously surviving these dangerous encounters leads to enjoyment (e.g., McCall et al. 2015; Zillmann and Weaver 1996). Indeed, actively overcoming negative emotions such as fear and anxiety is one of the main sources of enjoyment that comes from playing video games (Lynch and Martins 2015; Ventura et al. 2013). Because games often provide players with continuous threats, challenges and rewards, the emotional experience of fear, anger and enjoyment may 
oscillate so rapidly that players may report experiencing all of these emotions in a single play session (Barrett et al. 2007). Regardless of whether players respond to acute ingame threats with fear, hostility, or both, the intensity of their emotional experience will likely depend on their sense of presence in the virtual environment (Parsons and Rizzo 2008; Price et al. 2011). It is expected that players who generally do not experience a sense of in-game presence, are less likely to be emotionally influenced by threatening situations. Therefore, the first aim of this study is to investigate whether playing video games in VR elicits a stronger sense of presence and subsequently stronger emotional responses than playing those games on a TV. By comparing both psychological and physiological responses to VR and TV, the role of immersive media within the effects of frightening and violent video games on presence and players' fearful and hostile responses may be clarified.

\subsection{Immersion and presence}

Immersion and presence are both widely used to describe the process of being transported from reality to a virtual environment, but due to the multidisciplinary use of these concepts there is no clear consensus regarding their defining differences (Georgiou and Kyza 2017). Some researchers have argued that immersion is a psychological state of mind, reflecting the degree in which users believe they are present in a virtual environment (e.g., Witmer and Singer 1998). However, most scholars describe immersion as a characteristic of the medium or a product of that medium, i.e. media or its products can be more or less immersive, leading to a corresponding sense of immersion among its users. The medium of VR is regarded as more immersive than traditional 2D screens because VR can display a more "inclusive, extensive, surrounding and vivid illusion of reality to the senses of a human participant" (Slater and Wilbur 1997, p. 606). Similarly, immersion has been defined as the objective and measurable properties of a mediated environment (Bystrom et al. 1999; Nash et al. 2000). In the current study, immersion is regarded as a characteristic of the medium, whereas presence will be used to describe whether an individual believes to be transported in a virtual environment (e.g. Nash et al. 2000). As such, in line with Wirth et al. (2007) we conceptualize presence as "the experiential counterpart of immersion" (p. 496).

Television screens are still the most popular home appliance for entertainment media, including video games (e.g., Chang and Hwang 2020). Larger television screens are more immersive because they fill a greater percentage of the viewer's visual field and are thereby more likely to evoke presence, leading to stronger positive emotional responses (Lombard et al. 2000; Reeves and Nass 1996). Headmounted VR displays are more immersive than TV screens by providing a user-centric perspective and a stereoscopic visual field that is continuously modified by head position and rotation, thereby allowing a visual illusion of movement in real-time (Czernuszenko et al. 1997). As a result, VR causes stronger feelings of presence than TV screens (e.g., Cummings and Bailenson 2016; Hou et al. 2011; Wirth et al. 2007). Presence describes the degree in which people experience a sense of being spatially located in the virtual environment (e.g., Coxon et al. 2016; Lin 2017), and it has also been referred to as physical presence (Lee 2004) or spatial presence (Lombard and Ditton 1997). Studies on players' responses to game environments demonstrated that when players used more immersive technology, they experienced a stronger sense of presence than when players used less immersive technology (Alshaer et al. 2017; Persky and Blascovich 2008). One recent study on immersive games found that perceived sense of presence was higher in the headmounted VR condition compared to the desktop (monitor) condition (Pallavicini et al. 2019). Therefore, the following hypothesis was formulated:

H1: Playing a video game in VR will result in stronger feelings of presence than playing the same game on a TV.

\subsection{Immersive horror and fright}

Fear has been defined as a multidimensional emotional reaction that includes both immediate physiological responses, and ensuing cognitive responses, to perceived threatening stimuli (Lynch and Martins 2015). Fear is often accompanied by changes in physiology (e.g., sweaty palms, elevated heart rate, trembling), expression (e.g., wide eyes, cowering, higher pitched tone of voice), information processing (e.g., more susceptible to suggested protective actions), and specific action tendencies (e.g., to seek cover, avoid or flee), although none of these changes are vital to experiencing this emotion (Kivikangas 2018). Constructionist theories of emotions argue that fear is experienced as a result of conceptualizing sensory input (both from the environment and from within the body) with learned knowledge of this emotion category (Barrett 2017). Survival horror games often provide jump scares and other unexpected mediated threats to the player's autonomic nervous system. Since our hearts are innervated by branches of the autonomic nervous system, these threats will cause an immediate acceleration in heart rate (Cacioppo et al. 2000). Our primal survival system has no time to process whether these threats are real, as it instinctively adapts our physiology to the perceived threat (Lynch 2018). The influence of threats on the autonomic nervous system can also be indexed by heart rate variability (HRV). HRV actually provides information on two quantities: heart rate and its variability, providing multiple tools 
to assess individual differences in physiological responses to fear (Appelhans and Luecken 2006). After the initial physiological scare, self-reflections on these experiences are integrated with information from earlier experiences and conceptual knowledge (Barrett 2017). Thus, both immediate physiological responses (e.g., heart rate and heart rate variability) and subsequent affective appraisals (e.g., subjective survey assessments) are valid measures of the emotional experience of fear, and both will be applied in this study.

In survival horror games, the main protagonist controlled by the player is often under-armed and vulnerable while being confronted with horrific events and life-threatening situations (Lynch and Martins 2015). These games utilize players' constant alertness to imminent death as a primary fear tactic. Combined with other fear mechanics, survival horror games were found to evoke stronger fear responses than any other game genre (Lynch and Martins 2015; Perron 2004). Furthermore, playing a survival horror game in VR will cause perceptual threats to be perceived in a manner that more closely mirrors reality (Parsons and Rizzo 2008). If users feel present, their sensory channels primarily perceive input generated by the media technology, reinforcing the notion that incoming information is coherent and believable, making it harder to consciously control their emotional and physiological responses (Riva et al. 2007). Research has shown that feelings of presence may intensify existing effects of video games (Klimmt et al. 2010). Several researchers have even argued that presence may be a necessary mediator that allows real emotions to be stimulated by a virtual environment (Parsons and Rizzo 2008; Price et al. 2011). Specifically, research has shown that survival horror games elicited emotions such as fear, suspense, and anxiety through players' sense of presence. More than from the mere perception of being located in a scary virtual environment, players' sense of fear came from the perception of virtual activities actually happening to them (Lin 2017). Thus, VR will likely provide a strong sense of spatial presence in a scary virtual environment, simultaneously heightening the sense that potential monsters are really there with you, thereby increasing feelings of fear. Vicariously surviving these horrific experiences should also increase reported enjoyment afterwards (Lynch and Martins 2015).

In general, highly-arousing, negatively valenced emotions, such as fear, provide individuals with a fundamental motivation to remove or avoid the threat (Eder et al. 2013). If avoidance is our natural response to threatening stimuli, why would anyone enjoy video games that aim to frighten, shock, and horrify its players? According to the excitation transfer theory (Zillmann and Weaver 1996), our enjoyment of horror films comes from feelings of suspense and arousal that arise when deplorable events threaten liked protagonists (see also Martin 2019). The degree of physiological excitement builds up during these suspenseful scenes and transfers to a corresponding degree of euphoria after the threatening events are positively resolved. Others have argued that a positive resolution is not necessary and that sustaining physiological arousal may be enjoyable in itself, motivating viewers to continue watching frightening content, regardless of the outcome (Berlyne 1967; Tamborini 1991). Even others have argued that viewers' enjoyment of horror movies comes from mastering fears through vicariously watching characters deal with horrific events (Sparks and Sparks 2000). If we transpose these arguments to survival horror games, it seems that players may experience more enjoyment than viewers because unlike helplessly watching the protagonist, players experience the freedom to confront threats as they choose. Regardless of the enjoyment that comes from vicariously mastering fears, games provide enjoyment through mastery over challenging game situations (Vorderer et al. 2003). Moreover, higher physiological excitement while playing should transfer to more enjoyment when frightening threats are successfully mastered. Since VR should lead to a stronger sense of presence and therefore, more fear and physiological arousal, this should eventually lead to more reported enjoyment when players succeed in mastering the horrific challenges. Indeed, a recent study found that highly aroused players, with strong reported fear, reported the highest enjoyment while playing a VR horror game (Lin et al. 2018). Therefore the following hypotheses were formulated:

H2: Playing a survival horror game in VR will lead to a stronger sense of fear than playing the same game on a TV.

H3: The feeling of presence mediates the effect of VR on fear.

H4: The feeling of presence mediates the effect of VR on enjoyment.

\subsection{Immersive horror and hostility}

Survival horror games, similar to horror movies, often feature characters being attacked or physically injured by supernatural or unnatural elements in order to frighten or terrify audiences (Hoffner and Levine 2005). Although reactive fear is often linked to flight rather than fight, individuals can engage in preemptive defensive aggression when confronted with frightening threats (Simunovic et al. 2013). Previous research has shown that violent games can cause anger and hostility following exposure (e.g., Carnagey and Anderson 2005). Similarly, players of survival horror games might experience the game as violent and subsequently experience hostility following gameplay. Furthermore, research on the relation between aggression and immersion found that more immersive violent games (i.e. newer games) resulted in more aggressive feelings than less immersive (i.e. older) violent 
games (Ivory and Kalyanaraman 2007; Sherry 2001). Specifically, playing a violent video game on a more immersive virtual platform led to higher levels of hostility than playing a violent game on a less-immersive platform (Persky and Blascovich 2008). Similarly, players who experienced more presence in a violent game reported more anger and hostility (Lull et al. 2016; Tamborini et al. 2004). Thus, players' sense of presence may mediate the effect of immersive entertainment on feelings of hostility.

Furthermore, heightened physiological arousal influences hostile reactions by making appraisals that are central to anger more likely to occur (Frijda and Zeelenberg 2001). An explanation for the effect of arousal on hostility is offered by the elementary arousal model (Tannenbaum and Zillmann 1975), which states that arousal is a heightened, nonspecific drive state, that will heighten emotional responses with increased energy and intensity. Thus, compared to playing on a TV, playing a horror game in VR will likely provide a higher sense of presence and more arousal, both of which may heighten hostile responses.

H5: Playing a violent game in VR will result in more hostility than playing it on a TV.

H6: The experience of presence mediates the effects of VR on hostility.

\subsection{Current study}

In short, the current study reports on two conceptually similar experiments. The first experiment tested the effect of horrific VR and TV game experiences on presence (H1), fear (H2), hostility (H5) and the mediating effects of presence (H3, H4 and H6). The second experiment was focused on violent VR and TV experiences, and how VR affects presence (H1), hostility (H5), and the mediating effect of presence (H4 and $\mathrm{H} 6)$.

\section{Experiment 1}

\subsection{Method}

\subsubsection{Sample and procedure}

Sample sizes were estimated on the basis of 30 respondents per laboratory condition, which is generally adequate to conduct mediational analyses with effects of large size (Fritz and MacKinnon 2007). A total of 66 participants were recruited to take part in a 1-h laboratory experiment in exchange for research credits or a 10 euro gift card. All individuals were informed beforehand that the experiment involved playing a horror game on a PlayStation 4. There were three inclusion criteria: (1) Participants must be at least 18 years old; (2) they should have some experience with recent video game controllers, and (3) no experience with Resident Evil 7 (Capcom 2017). All participants provided active consent before participating. All participants ( $M$ age $=22.24, S D=2.49$ ) played the survival horror game Resident Evil 7, either on a TV or in VR. The majority of participants was male $(n=46 ; 69.7 \%)$, and most were students at the University of Amsterdam $(n=61)$. In the VR condition, seven female participants dropped out after approximately $10 \mathrm{~min}$ of play due to reported nausea. Data from these participants were not included in the analysis, leading to 59 participants being included in the final sample: 31 participants in the VR condition and 28 in the TV condition.

A pilot test was conducted to establish the number of scares during one play session. These scary moments were used as reference points for the physiological measurements and the subjective fright experiences. A pilot test with four participants indicated that there were five particularly scary moments during the play session, as indicated by elevated heart rate and subjective assessments from the participants. The effects of the following five scares were assessed: (1) complete darkness upon entering the house; (2) discovering the body of Andre, a news reporter; (3) a severed human head popping out of the water; (4) Mia, the protagonist's wife, crawling up the stairs and subsequent jump-scare; (5) Mia jumping to her feet and attacking after seeming unconscious. The moments when participants reached these five points in the game were marked during the experimental procedure for HRV analyses (see "Measures").

Each participant was randomly assigned to either the experimental group who played Resident Evil 7 (Capcom 2017) on the PlayStation VR or the control group who played the same game on a TV. In the VR condition, head tracking technology within PlayStation VR goggles allowed for a $360^{\circ}$ view, six degrees of freedom, and separate left and right images that allowed players to experience stereo vision. In the TV condition, the game was played on a 32 inch flat screen TV. In both conditions, the PS4 Dualshock controller was used to move the character and interact with the environment. Headphones provided directional audioeffects, which could help players identify the source and direction of events through sound.

The experiment consisted of four stages; pre-test questionnaire, relaxation, gaming, and post-test questionnaire. After completing the first part of the questionnaire, participants were aided with applying the Polar heart rate monitor, a rubber band strapped across their chest. If the computer registered a consistent heart rate, both groups started a new game, including the introduction. Participants played for 40-60 min until they reached the part where they 'killed' Mia and picked up a ringing telephone. All participants reached this part without dying. Researchers calmly entered the room, turned on the lights and helped 
them take off the VR headset or shut down the TV. Finally, participants completed the last part of the questionnaire on a laptop. Upon completing the final stage of the study, participants were thanked and debriefed.

\subsection{Measures}

\subsubsection{Presence}

Players' sense of presence was measured using seven items from the MEC Spatial Presence Questionnaire (Sacau et al. 2005). The 7-item scale measured to what extent the participant felt that they were an integral part of a plausible reality. All presence items were measured on a 1 (strongly disagree) to 5 (strongly agree) response scale. Sample items included 'I felt like I was actually in the environment of the game' and 'I felt as though I was in the same space as the characters and objects'. The scale was unidimensional explaining $65 \%$ of the variance. A mean score was calculated with higher scores reflecting, higher levels of presence. This 7-item scale proved reliable $(\alpha=0.91, M=3.45, S D=0.87)$.

\subsubsection{Hostility}

We used a selection of four items from the State Hostility Scale (Anderson and Carnagey 2009) to measure participants' current aggressive and aggravated state before and after playing the game. The four items: 'I feel furious', 'I feel aggravated', 'I feel angry', and 'I feel enraged' were measured on a 1 (strongly disagree) to 5 (strongly agree) response scale. This scale was unidimensional, explaining $55 \%$ of the variance. Feelings of hostility were reliably measured before play ( $\alpha=0.77, M=1.58, S D=0.64)$ and immediately after play ( $\alpha=0.73, M=1.84, S D=0.68)$. Change scores were calculated by subtracting participants' pre-test hostility scores from post-test hostility scores, in order to compare any hostility enhancing effects.

\subsubsection{Subjective fear}

Immediately after their play session, participants answered three self-reflection items about their experience of fear during the game, similar to items used by Lin (2017). All items were rated on a 10-point scale, ranging from 1 (not at all) to 10 (very much). Items were preceded by 'Please indicate the how you felt during the game': (1) 'I was frightened', (2) 'I was scared', and (3) 'I was afraid'. This scale proved reliable $(\alpha=0.92)$ and participants generally felt frightened during the game $(M=5.98, S D=2.14)$.

\subsubsection{Subjective enjoyment}

The positive emotional outcome of playing was measured using three items: 'I thought the game was enjoyable', 'I thought the game was exciting', and 'I thought the game was amusing'. All items were rated on a 5-point response scale, ranging from 1 (not at all) to 5 (very much). The enjoyment scale proved reliable $(\alpha=0.87, M=3.20, S D=1.02)$.

\subsubsection{Heart rate (HR) and heart rate variability (HRV)}

Heart rate (HR) is based on the number of contractions of the ventricles per minute. Heart rate variability (HRV) is significantly associated with HR, providing information on both the HR and its variability. HRV describes the physiological variation in the time interval between heartbeats. It is measured by the variation in the beat-to-beat interval. In general, lower HRV indicates physiological or psychological stress. The Polar S810 chest-band HRM was used to gather HRV data. Previous research has indicated that this device can accurately assess HRV in healthy subjects (Gamelin et al. 2006). The method used to analyse HRV is known as RMSSD: the square root of the mean squared difference between adjacent normal to normal intervals (Wang et al. 2009). During the gameplay session, five scare moments were manually marked by the researchers when encountered by the players. HRV for the subsequent $20 \mathrm{~s}$ was calculated. All HRV values of 0.000 were set to missing due to measurement error ( $n=5 ; 12$ out of 295 cells). HRV scores for 56 participants could be computed and used for analyses. Mean HRV across the five 20-s intervals were used to compute the total mean HRV for each player $(n=56$, range 2.42-203.68; $M=62.11, S D=42.50$ ).

\section{Results and discussion}

The first hypothesis stated that the immersive qualities of VR would lead to a stronger sense of presence. Indeed, the extent to which participants felt they were actively taking part in a plausible reality, was stronger among VR players $(M=3.83, S D=0.69)$ than among TV players $(M=3.03$, $S D=0.87), t(57)=3.94, p<0.001, d=1.02$. Although players' emotional responses were generally stronger in the VR condition, $t$ tests showed no significant differences in emotional responses of participants in the VR condition (Fear $M=6.20, S D=2.27$; Enjoyment $M=3.78, S D=0.97$; Post-play Hostility $M=1.91, S D=0.71)$ compared to the TV condition (Fear $M=5.74, S D=2.00$; Enjoyment $M=3.00$, $S D=1.06$; Post-play Hostility $M=1.78, S D=0.66$ ), all $p \mathrm{~s}>0.16$.

We found a strong correlation between participants heart rate (HR) and their self-reported fear $(r=0.42, p=0.001)$, 
and the relation between heart rate variability (HRV) and subjective fear was borderline non-significant $(r=-0.24$, $p=0.065$ ). Since no significant correlations between HR and hostility, nor HRV and hostility were found, it seems that players' physiological stress responses are best described as indicators of fearful emotions. Regardless of experimental condition, participants reported an increase in feelings of hostility from pre-play $(M=1.58, S D=0.64)$ to post-play $(M=1.84, S D=0.68, t(58)-3.16, p=0.003)$. However, this increase in hostility between pre- and post-play $(M=0.27$, $S D=0.65)$ did not differ between VR $(M=0.36, S D=0.70)$ and TV $(M=0.16, S D=0.58), t(57)=1.20, p=0.230$.

We found some post hoc gender differences in the experience of playing this survival horror game. Overall, female players reported more fear while playing $(M=7.03$, $S D=1.49)$ than male players did $(M=5.68, S D=2.22), t$ (28.66) $=-2.55, p=0.022, d=0.71$. Furthermore, male participants reported more enjoyment in the VR condition $(M=3.60, S D=0.64)$ as compared to the TV condition $(M=3.04, S D=1.92), t(43) 2.22, p=0.034, d=0.67$, whereas female participants did not.

The physiological differences in HR and HRV between VR and TV conditions across the five scare moments in the survival horror experiment are displayed in Fig. 1. Participants who played in VR showed a significantly lower mean HRV across the five scare moments $(M=49.76, S D=29.15)$ than those who played on a TV $(M=74.47, S D=50.15)$, $t$ (43.38) 2.26, $p=0.038, d=0.60$. Although HR was generally higher when playing in VR $(M=80.14, S D=16.78)$ than on a TV $(M=74.62, S D=18.42)$, this difference was not significant, $t$ (56) $1.19, p=0.238$. Since lower HRV is an indicator of physiological stress, it is assumed that VR causes more physiological stress than TV because of the higher amounts of fear experienced by players.

To test whether there was an indirect relationship between immersive technology (TV versus VR) via presence on physiological and emotional responses, the PROCESS macro for SPSS (Hayes 2012) was used to conduct a parallel mediation analysis using 5000 bootstrap samples. First, the independent variable (TV versus VR) was regressed on HRV. This first model with HRV as dependent variable was significant, $R=0.47, R^{2}=0.23, F(1$, $54)=15.93, p<0.001$. The VR condition had a significant total effect on HRV, $B=-20.17, t=-2.04, p=0.04$. As expected, we found near-identical significant effects of VR on the reported sense of presence across all models (HRV, Hostility, Fear, and Enjoyment). The general mediation model is displayed in Fig. 2, and all corresponding mediation path analyses are summarized in Table 1. VR significantly influenced presence, $\beta=0.95, t=3.99, p<0.001$, which subsequently affected HRV, $\beta=-0.31, t=-2.16$, $p=0.040$. This model shows that the VR condition of this

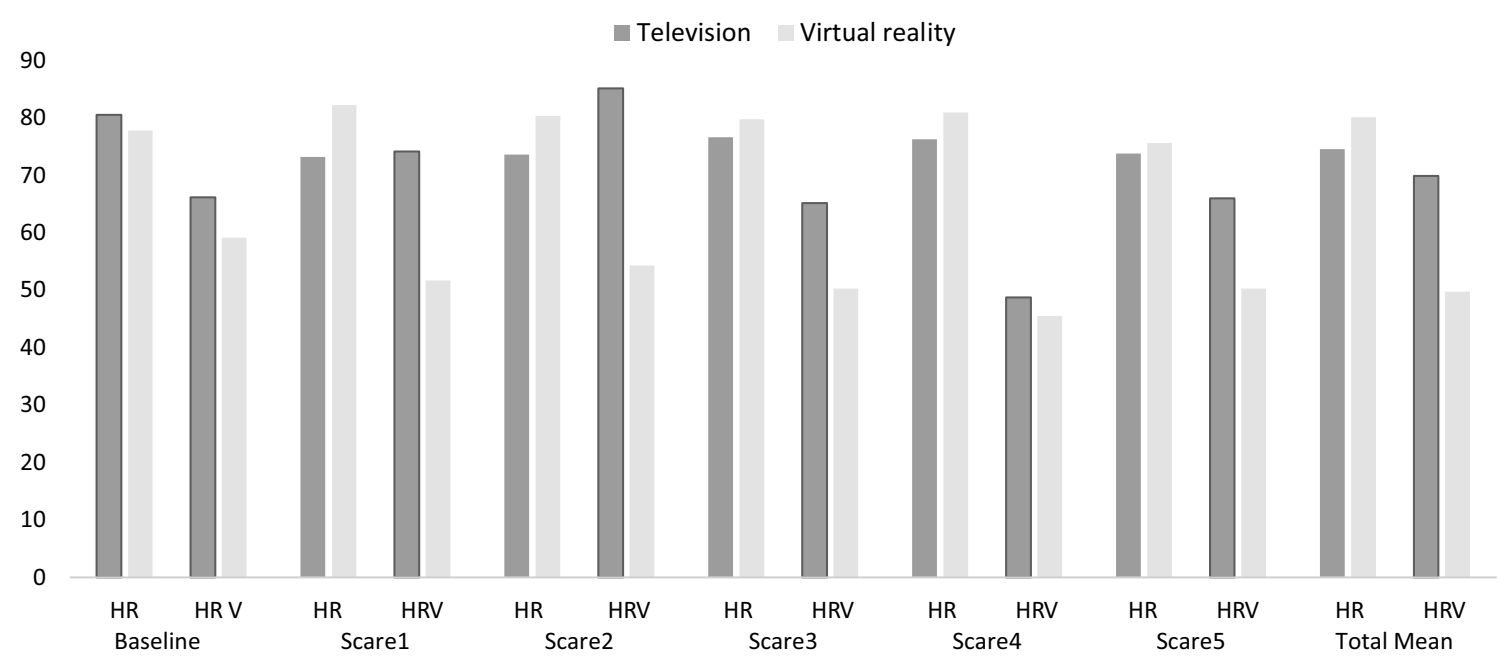

Fig. 1 Overview of mean heart rate and HRV between TV and VR conditions

Fig. 2 Process model: VR influences dependent variable by increasing presence d

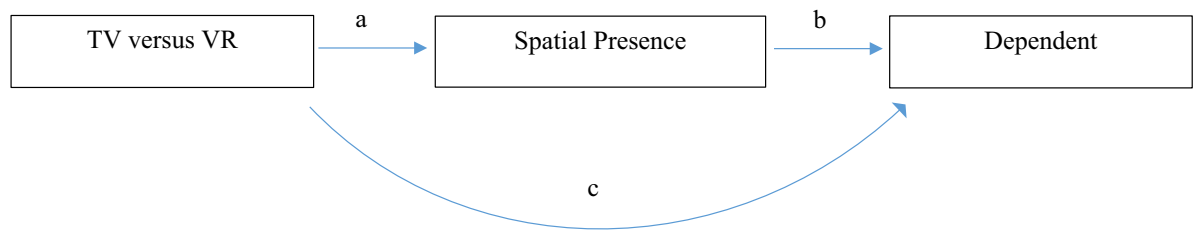


Table 1 Total effects, direct effects, mediation effects and indirect effects of all relevant variables

\begin{tabular}{|c|c|c|c|c|c|c|c|c|c|c|c|}
\hline \multirow[t]{3}{*}{ Dependent variables } & \multicolumn{11}{|c|}{ Experiment 1} \\
\hline & \multicolumn{2}{|c|}{ Total effect (d) } & \multicolumn{2}{|c|}{ Direct effect (c) } & \multicolumn{2}{|c|}{$\begin{array}{l}\mathrm{VR} \rightarrow \text { Presence } \\
\text { (a) }\end{array}$} & \multicolumn{2}{|c|}{ Presence $\rightarrow$ D (b) } & \multicolumn{3}{|c|}{ Indirect effect $(a+b-c)$} \\
\hline & $b$ & SE & $b$ & SE & $b$ & SE & $b$ & SE & $b$ & Boot SE & LLCI, ULCI \\
\hline HRV & $-20.17 *$ & 9.86 & -8.99 & 10.86 & $0.85 * * *$ & 0.21 & $-13.20 *$ & 6.12 & $-11.18^{*}$ & 5.61 & $(-23.32,-1.47)$ \\
\hline Fear & 0.46 & 0.56 & -0.62 & 0.56 & $0.80 * * *$ & 0.20 & $1.34 * * *$ & 0.32 & $1.07 *$ & 0.38 & $(0.40,1.88)$ \\
\hline Hostility & 0.20 & 0.17 & 0.05 & 0.19 & $0.80 * * *$ & 0.20 & 0.18 & 0.11 & $0.15^{*}$ & 0.08 & $(0.01,0.32)$ \\
\hline Enjoyment & 0.38 & .27 & 0.25 & 0.30 & $0.80 * * *$ & 0.21 & 0.17 & 0.17 & 0.13 & 0.16 & $(-0.19,0.46)$ \\
\hline \multirow[t]{3}{*}{ Dependent variables } & \multicolumn{11}{|c|}{ Experiment 2} \\
\hline & \multicolumn{2}{|c|}{ Total effect (d) } & \multicolumn{2}{|c|}{ Direct Effect (c) } & \multicolumn{2}{|c|}{$\begin{array}{l}\text { VR } \rightarrow \text { Presence } \\
\text { (a) }\end{array}$} & \multicolumn{2}{|c|}{ Presence $\rightarrow$ D (b) } & \multicolumn{3}{|c|}{ Indirect effect $(a+b-c)$} \\
\hline & $b$ & SE & $b$ & SE & $b$ & SE & $b$ & SE & $b$ & Boot SE & LLCI, ULCI \\
\hline HRV & -3.33 & 5.35 & -2.17 & 5.71 & $0.57 * *$ & 0.22 & -2.04 & 3.34 & -1.16 & 2.22 & $(-6.13,3.08)$ \\
\hline Hostility & -0.14 & 0.14 & -0.26 & 0.14 & $0.64 * * *$ & 0.20 & $0.19 *$ & 0.08 & $0.12 *$ & 0.07 & $(0.00,0.28)$ \\
\hline Enjoyment & -0.40 & 0.28 & $-0.70^{*}$ & 0.29 & $0.64 * * *$ & 0.20 & $0.47 * *$ & 0.17 & $0.30 *$ & 0.15 & $(0.05,0.65)$ \\
\hline
\end{tabular}

${ }^{* * *} p<.001 ; * * p<.01 ; * p<.05$

horror game had both a direct effect on lowering HRV and an indirect effect on lowering HRV through an enhanced sense of presence. Presence was negatively related to HRV, indicating an increase in players' physiological stress response.

The second model including fear as the dependent variable was also significant, $R=0.46, R^{2}=0.21, F(1,57)=15.52$, $p<0.001$. Again, the VR condition significantly influenced presence, $\beta=0.92, t=3.94, p<0.001$, and presence influenced fear, $\beta=0.55, t=4.17, p<0.001$. There was no significant total or direct effect of VR on fear, but there was a strong indirect effect through players' sense of presence on fear, $\beta=0.50,95 \% \mathrm{CI}[0.20,0.84]$. The third model included the increase in hostility between pre- and post-game measurements as dependent variable. This model was not significant, $R=0.15, R^{2}=0.02, F(1,57)=1.43, p=0.234$. Table 1 shows the effect of VR on presence, $\beta=0.92, t=3.94$, $p<0.001$. No total or direct effects for VR on hostility were found. However, there was a small indirect effect through presence, $\beta=0.22,95 \%$ [0.0044, 0.4858].

The final model examined whether presence in VR would also lead to more reported enjoyment. This model was not significant, $R=0.22, R^{2}=0.05, F(2,54)=1.45, p=0.252$. No total, direct, or indirect effects were found, indicating that despite the higher sense of presence, playing a horror game is not more enjoyable in VR than playing on TV $(H 4)$. In short, these four mediation models showed that players of a horror game in VR reported higher levels of presence (HI) and displayed more physiological stress than those who played on a TV screen. This increase in stress is likely caused by players' heightened emotional fear (H2) resulting from the immersive qualities of the medium, which made them feel more present in the game's environment $(H 3)$.

\section{Experiment 2}

\subsection{Sample and procedure}

Because of the relatively high dropout rate of female participants $(n=7)$ in the first experiment, only male participants $(N=71)$ were recruited for the second VR experiment. In line with Experiment 1, the study was conducted among young adults who were instructed to play a video game either in VR or on a large TV screen. Two subjects from the VR condition dropped out due to severe nausea after approximately ten minutes of play. Their (partial) responses were not included in the analyses. Data from 69 participants were included: 33 participants in the VR condition and 36 participants in the TV condition, $M$ age $=22.75, S D=4.91$.

Rather than playing a survival horror game which was expected to trigger fear and to a lesser extent hostility, participants in "Experiment 2" played a first-person shooter which was expected to trigger hostility. Threats to survival in first-person shooters (FPS) generally do not encourage fearful responses in players, but challenge them to rapidly react to threats by shooting and killing (e.g., Deluze et al. 2017; Colzato et al. 2010). In line with Experiment 1, participants were randomly assigned to either the experimental group who played Doom VR (Bethesda 2017) on the PlayStation VR or the control group who used the PS4 to play Doom (Bethesda 2016) on a TV. Participants used 
a controller to interact with the game, and earphones to experience audio. After providing consent, subjects were assisted in applying the Polar heart rate monitoring strap around their chest. Before playing, participants completed a small online survey about their demographics, experience and familiarity with first-person shooters, virtual reality gaming, and their current state of hostility. The experimenters took a few minutes to explain the game mechanics to both groups, and provided a brief demonstration on how to use the controller to move, shoot, switch weapons, perform glory kills, or teleport (only in the VR condition). After playing, participants were assisted with removal of the Polar strap and VR headset (if applicable) and were instructed to complete the post-test survey. Upon completion, subjects were thanked and debriefed.

\subsection{Measures}

\subsubsection{Hostility}

We used a selection of seven items from the State Hostility Scale (Anderson and Carnagey 2009) to measure participants' current aggressive and aggravated state before and after playing the game. The seven items: 'I feel aggressive', 'I feel mean', 'I feel stormy'. 'I feel furious', 'I feel aggravated', 'I feel angry', and 'I feel enraged' were measured on a 1 (strongly disagree) to 5 (strongly agree) response scale. This 7 -item scale was unidimensional, explaining $60-67 \%$ of the variance. Feelings of hostility were reliably measured before play, $\alpha=0.91, M=1.30$, $S D=0.51$, and after play, $\alpha=0.88, M=1.65, S D=0.72$. Change scores were calculated by subtracting participants' pre-test hostility scores from post-test hostility scores, in order to compare any hostility enhancing effects.

\subsubsection{Presence}

Players' sense of presence was assessed with items from the MEC Spatial Presence Questionnaire (Sacau et al. 2005) and the spatial presence subscale from the ITC Sense of Presence Inventory (Lessiter et al. 2001). The seven selected items differed slightly from those in the first experiment. All presence items were measured on a 1 (strongly disagree) to 5 (strongly agree) response scale. Sample items included 'I had the sense of being in the displayed environment' and 'I had the sensation that the characters were aware of me'. The scale was unidimensional explaining $55 \%$ of the variance. A mean score was calculated with higher scores reflecting higher levels of presence. This 7 -item scale proved reliable, $\alpha=0.86$, $M=3.42, S D=0.88$.

\subsubsection{Enjoyment}

The positive emotional outcome of playing was measured using three items: 'I thought the game was enjoyable', 'I thought the game was pleasurable', and 'I thought the game was amusing'. All items were rated on a 5-point response scale, ranging from 1 (not at all) to 5 (very much). The enjoyment scale proved reliable, $\alpha=93, M=3.43, S D=1.18$.

\subsubsection{Heart rate variability}

In line with Experiment 1, heart rate variability (HRV) was measured using the Polar S810 chest-band HRM. Manual markings were used to delineate participants' HRV intervals $20 \mathrm{~s}$ before gameplay while completing the pre-test survey (i.e. baseline) and for $20 \mathrm{~s}$ at three minutes before the end of gameplay. Similar to Experiment 1, HRV values of 0.000 caused by measurement errors $(n=8)$ were set to missing. The mean HRV during gameplay was 58.42, $S D=29.52$, and the mean heart rate (HR) was 78.54, $S D=12.60$.

\section{Results and discussion}

In line with Experiment 1, playing in VR had a significant influence on players' sense of presence. Players reported a stronger sense of spatial presence in VR $(M=3.76$, $S D=0.84)$ than when they played on a TV $(M=3.12$, $S D=0.81), t(67) 3.23, p=0.002, d=0.78$. Contrary to the male players from experiment 1 , there was no significant difference in reported enjoyment between VR $(M=3.22$, $S D=1.22)$, and TV $(M=3.62, S D=1.13), t(67) 1.41$, $p=0.164$. Regardless of condition, players reported a small increase in hostility when comparing scores before playing $(M=1.29, S D=0.53)$ and after playing $(M=1.58$, $S D=0.73), t(68)=-4.40, p<0.001$. However, the increase in hostility between pre- and post-play measures $(M=0.27$, $S D=0.57)$ did not differ between VR $(M=0.36, S D=0.70)$ and TV $(M=0.16, S D=0.58), t(57)=-1.20, p=0.236$. These results indicate that although VR leads to a stronger sense of presence, it does not directly influence hostile responses to that environment.

To test whether there was an indirect relationship between immersive technology (TV versus VR) via presence on physiological and emotional responses, the PROCESS macro for SPSS (Hayes 2012) was used to conduct a parallel mediation analysis using 5,000 bootstrap samples. Similar to the first experiment, we found significant effects of VR on the reported sense of presence across all included dependent variables (HRV, Hostility, and Enjoyment). However, the overall parallel mediation model with HRV as dependent variable was not significant, $R=0.12, R^{2}=0.01$, $F(1,55)=0.38, p=0.68$. In this model, VR significantly 
affected presence $(\beta=0.66, t=2.63, p=0.01)$, but presence did not predict HRV $(\beta=-0.09, t=-0.61, p=0.54)$ and no direct, total or indirect effects were found (see Table 1).

Similarly, the parallel mediation model including the change between pre- and post-game hostility as dependent variable was also not significant, $R=0.12, R^{2}=0.02, F$ $(1,67)=1.02, p=0.31$. VR again showed a significant effect on presence $(\beta=0.73, t=3.23, p=0.001)$, and presence significantly influenced the pre- to post-game change in hostility, $\beta=0.29, t=2.26, p=0.03$, but the indirect effect of VR through presence on hostility was not significant, $\beta=0.21$, $95 \%$ CI $[-0.001,0.49]$. Finally, the model with enjoyment as dependent variable was also not significant, $R=0.17$, $R^{2}=0.03, F(1,67)=1.98, p=0.16$. As before, VR predicted presence $(\beta=0.73, t=3.23, p=0.001)$, but there was a negative effect of VR on enjoyment $(\beta=-0.59, t=-2.24$, $p=0.02$ ), and a positive indirect effect through presence, $\beta=0.25,95 \%$ CI $[0.0488,0.6486]$.

In general, this second experiment again showed that playing in VR leads to a stronger sense of presence in the virtual environment than playing on a TV. Feelings of hostility increased overall during play, but no differences between VR and TV emerged (H5). Furthermore, the indirect effect of VR on hostility through presence was not significant (H6). Contrary to the survival horror game from the first experiment, the male participants in this experiment did not enjoy the VR version of the FPS more than the TV version. This null-effect on enjoyment may be related to the fact that although the VR and TV versions of the game came from the same franchise with similar narratives and graphics, their game mechanics were vastly different. The controls for Doom VR proved somewhat difficult, resulting in the procedure being interrupted for many participants in order to provide a few quick tips. These difficulties in navigating the game may have resulted in somewhat lower enjoyment in the VR condition.

\section{General discussion}

The main aim of our study was to examine whether playing commercially available games in VR would result in a stronger sense of presence and stronger emotional and physiological responses than playing those same games on a TV. In line with our expectations, VR provided a more immersive experience than playing on a TV. A stronger sense of spatial presence was observed for both the horror survival game and the first-person shooter. Based on observations by Slater (2009) and Skarbez et al. (2017), we argue that players of virtual reality games experience a strong sense of being transported to a virtual place that seems plausible and consistent with their expectations, causing players to respond in ways that are similar to their responses if these situations were real. Our second aim was to examine whether playing games in VR would result in stronger emotional responses than playing on a TV. Results from the first experiment indicated indirect effects of VR on both fear and hostility through players sense of presence in the game environment. These indirect relationships underline the important role of feeling present in the virtual environment within the experience of fear and hostility. In line with previous studies (Parsons and Rizzo 2008; Price et al. 2011), we agree that presence mediates the effectiveness of game content on emotional responses.

These findings are mirrored by the results from the physiological measures. The first experiment showed that playing in VR resulted in lower heart rate variability than playing on a TV. This decrease in heart rate variability (HRV) might best be viewed as an indicator of psychological fear-related stress caused by this immersive horror game. Mediation analyses indicated that VR had a direct effect on HRV and that this effect was partially mediated by players' sense of presence. Although lower HRV can also be an indicator of increased reactive aggression (Scarpa et al. 2010), it seems more likely that lower HRV displayed by players of the survival horror game is actually the physiological response to feelings of fear. This study thereby emphasizes the need to distinguish the theoretical roles of HR and HRV within research on the emotional effects of immersive media.

Physiological arousal is not only considered a central component of emotional experiences and an important affective enhancement mechanism, it also increases attentional awareness (e.g., Kühne 2012). According to the arousal theory of presence (Freeman et al. 2005) arousal leads to alertness, which could lead to greater perceived physical and mental presence in VR. Alternatively, Diemer et al. (2015) argue that emotionally relevant stimuli and information (e.g., horror and violence) make VR environments more interesting and appealing to attention (suggesting an increase in presence), which would increase arousal. Although the consistent relations between physiological arousal (increased HR and decreased HRV) and presence found in our studies could support these assumptions, we argue that the immersiveness of VR, and not arousing content, is the primary reason for players' sense of presence. In line with conclusions by Lin (2017), we find that VR leads to a stronger sense of presence than a less immersive medium (2D screen), and the stronger sense of being present in scary or violent environments leads to corresponding physiological arousal (increased HR and decreased HRV) and emotional responses. Future research could perhaps also include less arousing environments and corresponding emotions and test alternative models to determine the precise relations between presence, physiological arousal and emotions in VR. 
Since VR provided a unique and immersive experience for many participants, we expected that this would result in an increased enjoyment, either because it provided a stronger sense of presence, or simply because of the 'novelty effect', since most participants had no experience with virtual reality (Bardo et al. 1996). However, we found that playing in VR generally did not result in more enjoyment than playing on a TV, with the exception of male participants in the first experiment. Male participants who played the survival horror game reported more enjoyment and more fear, when comparing VR to TV, than female participants. When frightening events lead to anxiety instead of excitement, enjoyment suffers (Sparks and Sparks 2000). Individuals have the ability to reappraise anxiety into excitement when they can detach themselves from harm by adopting a frame of mind where the observed experience does not present any real danger (Andrade and Cohen 2007). Obviously, playing a horror game does not present any real danger to the player, but the higher sense of presence in VR may cause more trouble adopting a frame of mind that allows for detachment from harm, thereby causing anxiety instead of excitement for some players. In general, men enjoy frightening and violent entertainment more than women do (e.g., Hoffner and Levine 2005). Since VR increased players' sense of fear, this may have decreased female enjoyment of VR considerably, which would explain why only men enjoyed the VR version of this game more than the TV version. Moreover, earlier research has shown that in order to enjoy frightening entertainment, a certain belief in his or her capacity to overcome the frightening events is required. Specifically, players with high self-efficacy, high arousal and severe fear, reported the highest enjoyment while playing a VR horror game (Lin et al. 2018). Thus, in future research it will be important to consider the level of self-efficacy and ability to successfully navigate the challenges provided in a game as moderators of the relationship between fear and enjoyment.

Although the second experiment showed that VR was responsible for a stronger sense of presence than TV, we did not find differences in heart rate, hostility nor enjoyment among our all-male sample when comparing the two conditions. Regardless of immersiveness, feelings of hostility went up after playing Doom, echoing conclusions from a study by Ivory and Kalyanaraman (2007), who also found increased hostility after exposure, but no effect of immersiveness on hostility. It is possible that players hostile responses were affected by differences in the games' formats. For instance, compared to the time-tested running and sidestepping mechanics of the original Doom, the complicated teleportation mechanics of Doom VR may have lessened players' engagement and thereby its emotional impact. Similarly, these differences in game format may have affected player enjoyment. In both studies, regardless of game genre, hostility increased significantly post-play, adding to the line of research suggesting short-term effects of violent games on hostility (e.g., Anderson and Bushman 2001; Ivory and Kalyanaraman 2007). However, contrary to previous studies (e.g., Tamborini et al. 2004), this study did not find an effect of presence on hostility after playing a violent game.

Apart from the limited technical possibilities to customize console headsets like the PlayStation VR to the player, even high-end VR headsets might be problematic for first time users. Unexperienced players will likely experience usability issues which may juxtapose the intended effects. For this reason, we recommend either to include an extended training session as part of an experiment or to use immersive experiences with intuitive interactions. Larger sample sizes are also recommended. Although the achieved power level proved to be adequate to detect moderate to large effect sizes in our sample from study 1 , a similar-sized sample may not have been enough to detect small to medium effect sizes in mediation analyses. Considering the general prevalence of small sample sizes in VR research, we highly recommend future studies to conduct a-priori sensitivity tests to determine the required effect size of interest based on sample size (Perugini et al. 2018). Furthermore, it is important to replicate and explore current VR studies when participants' experiences with VR have become more common. It will be important to evaluate how adaptation of this technology will influence research in this area.

In conclusion, we believe that this study is an important step in this emerging research area. VR gaming is a highly immersive technology that enhances feelings of presence which influence the physiological and emotional state of its players. VR games allow players to experience vivid threats that feel identical to reality, causing players to instinctively adapt their physiology to the perceived threat. Presence thereby mediates the effectiveness of game content on physical and emotional responses. VR games allow real-time responses to dynamic changes in the virtual environment, and they can subject players to truly terrifying experiences. If you want media entertainment that provides strong physiological and emotional responses, buy the ticket, take the ride (Thompson 1971).

Open Access This article is licensed under a Creative Commons Attribution 4.0 International License, which permits use, sharing, adaptation, distribution and reproduction in any medium or format, as long as you give appropriate credit to the original author(s) and the source, provide a link to the Creative Commons licence, and indicate if changes were made. The images or other third party material in this article are included in the article's Creative Commons licence, unless indicated otherwise in a credit line to the material. If material is not included in the article's Creative Commons licence and your intended use is not permitted by statutory regulation or exceeds the permitted use, you will need to obtain permission directly from the copyright holder. To view a copy of this licence, visit http://creativecommons.org/licenses/by/4.0/. 


\section{References}

Alghamdi M, Regenbrecht H, Hoermann S, Swain N (2017) Mild stress stimuli built into a non-immersive virtual environment can elicit actual stress responses. Behav Inf Technol 36(9):913-934

Alshaer A, Regenbrecht H, O'Hare D (2017) Immersion factors affecting perception and behaviour in a virtual reality power wheelchair simulator. Appl Ergon 58:1-12

Anderson CA, Carnagey NL (2009) Causal effects of violent sports video games on aggression: is it competitiveness or violent content? J Exp Soc Psychol 45:731-739

Andrade EB, Cohen JB (2007) On the consumption of negative feelings. J Consum Res 34:283-300

Appelhans BM, Luecken LJ (2006) Heart rate variability as an index of regulated emotional responding. Rev Gen Psychol 10:229-240

Bardo MT, Donohew RL, Harrington NG (1996) Psychobiology of novelty seeking and drug seeking behavior. Behav Brain Res $77: 23-43$

Barrett LF (2017) The theory of constructed emotion: an active inference account of interoception and categorization. Soc Cognit Affect Neurosci 12(1):1-23

Barrett LF, Mesquita B, Ochsner KN, Gross JJ (2007) The experience of emotion. Annu Rev Psychol 58:373-403

Botella C, Serrano B, Baños RM, Garcia-Palacios A (2015) Virtual reality exposure-based therapy for the treatment of post-traumatic stress disorder: a review of its efficacy, the adequacy of the treatment protocol, and its acceptability. Neuropsychiatr Dis Treat $11: 25-33$

Bystrom KE, Barfield W, Hendrix C (1999) A conceptual model of the sense of presence in virtual environments. Presence Teleoper Virtual Environ 8:241-244

Cacioppo JT, Berntson GG, Larsen JT, Poehlmann KM, Ito TA (2000) The psychophysiology of emotion. Hand Emot 2:173-191

Cardo RA, David OA, David DO (2017) Virtual reality exposure therapy in flight anxiety. Comput Hum Behav 72:371-380

Carnagey NL, Anderson CA (2005) The effects of reward and punishment in violent video games on aggressive affect, cognition, and behavior. Psychol Sci 16(11):882-889

Chang J, Hwang J (2020) The role of media in user participation: Focusing on the knowledge activity in online space. Telemat Inf 51:101407

Coxon M, Kelly N, Page S (2016) Individual differences in virtual reality: are spatial presence and spatial ability linked? Virtual Real 20:203-212

Cummings JJ, Bailenson JN (2016) How immersive is enough? A meta-analysis of the effect of immersive technology on user presence. Media Psychol 19:272-309

Czernuszenko M, Pape D, Sandin D, DeFanti T, Dawe GL, Brown MD (1997) Immersa desk and infinity wall projection-based virtual reality displays. Comput Graph 31:46-49

Diemer J, Alpers GW, Peperkorn HM, Shiban Y, Mühlberger A (2015) The impact of perception and presence on emotional reactions: a review of research in virtual reality. Front Psychol 6:26

Diemer J, Lohkamp N, Mühlberger A, Zwanzger P (2016) Fear and physiological arousal during a virtual height challenge-effects in patients with acrophobia and healthy controls. J Anxiety Disord 37:30-39

Emmelkamp PM, Meyerbröke K, Morina N (2020) Virtual reality therapy in social anxiety disorder. Curr Psychiatry Rep 22(7):32

Freeman J, Lessiter J, Pugh K, Keogh E (2005) When presence and emotion are related, and when they are not. In: 8th annual international workshop on presence, pp 21-23

Fritz MS, MacKinnon DP (2007) Required sample size to detect the mediated effect. Psychol Sci 18(3):233-239
Gamelin FX, Berthoin S, Bosquet L (2006) Validity of the polar S810 heart rate monitor to measure RR intervals at rest. Med Sci Sports Exerc 38:887-893

Georgiou Y, Kyza EA (2017) The development and validation of the ARI questionnaire: an instrument for measuring immersion in location-based augmented reality settings. Int J Hum Comput Stud 98:24-37

Hayes AF (2012) PROCESS: a versatile computational tool for observed variable mediation, moderation, and conditional process modeling [White paper]. Retrieved from http://www.afhayes.com/ public/process2012.pdf

Hoffner CA, Levine KJ (2005) Enjoyment of mediated fright and violence: a meta-analysis. Media Psychol 7:207-237

Ivory JD, Kalyanaraman S (2007) The effects of technological advancement and violent content in video games on players' feelings of presence, involvement, physiological arousal, and aggression. J Commun 57:532-555

Kivikangas JM (2018) Emotion theories, the affective system, and why a digital games researcher should care. In: Breuer J, Pietschmann D, Liebold B, Lange BP (eds) Evolutionary psychology and digital games: digital hunter-gatherers. Routledge, Abingdon

Klimmt C, Hefner D, Vorderer P, Roth C, Blake C (2010) Identification with video game characters as automatic shift of self-perceptions. Media Psychol 13:323-338

Kühne R (2012) Media-induced affects and opinion formation: How related and unrelated affects influence political opinions. Living Rev Democr 3:1-20

Lee KM (2004) Presence, explicated. Commun Theory 14:27-50

Lessiter J, Freeman J, Keogh E, Davidoff J (2001) A cross-media presence questionnaire: the ITC-sense of presence inventory. Teleoper Virtual Environ 10:282-297

Lin JHT (2017) Fear in virtual reality (VR): fear elements, coping reactions, immediate and next-day fright responses toward a survival horror zombie virtual reality game. Comput Hum Behav 72:350-361

Lin JHT, Wu DY, Tao CC (2018) So scary, yet so fun: the role of selfefficacy in enjoyment of a virtual reality horror game. New Media Soc 20:3223-3242

Lombard M, Ditton T (1997) At the heart of it all: the concept of presence. J Comput Med Commun 3(2):JCMC321. https://doi.org/10. 1111/j.1083-6101.1997.tb00072.x

Lombard M, Reich RD, Grabe ME, Bracken CC, Ditton TB (2000) Presence and television. The role of screen size. Hum Commun Res 26:75-98

Lynch T (2018) Evolutionary formidability mechanisms as moderators of fear experience. In: Breuer J, Pietschmann D, Liebhold B, Lange BP (eds) Evolutionary psychology and digital games: digital hunter-gatherers. Routledge, London

Lynch T, Martins N (2015) Nothing to fear? An analysis of college students' fear experiences with video games. J Broadcast Electron Media 59:298-317

Martin GN (2019) (Why) do you like scary movies? A review of the empirical research on psychological responses to horror films. Front Psychol 10:2298

McCall C, Hildebrandt LK, Bornemann B, Singer T (2015) Physiophenomenology in retrospect: memory reliably reflects physiological arousal during a prior threatening experience. Conscious Cogn 38:60-70

Nash EB, Edwards GW, Thompson JA, Barfield W (2000) A review of presence and performance in virtual environments. Int J Hum Comput Interact 12:1-41

Pallavicini F, Pepe A, Minissi ME (2019) Gaming in virtual reality: what changes in terms of usability, emotional response and sense of presence compared to non-immersive video games? Simul Gaming 50:136-159 
Parsons TD, Rizzo AA (2008) Affective outcomes of virtual reality exposure therapy for anxiety and specific phobias: a meta-analysis. J Behav Ther Exp Psychiatry 39:250-261

Perron B (2004) Sign of a threat: the effects of warning systems in survival horror games. In: COSIGN 2004 Proceedings, pp 132-141

Persky S, Blascovich J (2008) Immersive virtual video game play and presence: influences on aggressive feelings and behavior. Presence Teleoper Virtual Environ 17:57-72

Perugini M, Gallucci M, Costantini G (2018) A practical primer to power analysis for simple experimental designs. Int Rev Soc Psychol 31:1

Price M, Mehta N, Tone EB, Anderson PL (2011) Does engagement with exposure yield better outcomes? Components of presence as a predictor of treatment response for virtual reality exposure therapy for social phobia. J Anxiety Disord 25:763-770

Reeves B, Nass C (1996) The media equation: How people treat computers, television, and new media like real people. Cambridge University Press, Cambridge

Riva G, Mantovani F, Capideville CS, Preziosa A, Morganti F, Villani $\mathrm{D}$ et al (2007) Affective interactions using virtual reality: the link between presence and emotions. Cyberpsychol Behav 10:45-56

Sacau A, Laarni J, Ravaja N, Hartmann T (2005) The impact of personality factors on the experience of spatial presence. In: The 8th international workshop on presence (Presence 2005), pp 143-151

Scarpa A, Haden SC, Tanaka A (2010) Being hot-tempered: autonomic, emotional, and behavioral distinctions between childhood reactive and proactive aggression. Biol Psychol 84:488-496

Sherry JL (2001) The effects of violent video games on aggression. Hum Commun Res 27:409-431

Skarbez R, Brooks FP Jr, Whitton MC (2017) A survey of presence and related concepts. ACM Comput Surv CSUR 50:1-39

Slater M (2009) Place illusion and plausibility can lead to realistic behaviour in immersive virtual environments. Philos Trans R Soc B Biol Sci 364:3549-3557

Slater M, Wilbur S (1997) A framework for immersive virtual environments (FIVE): speculations on the role of presence in virtual environments. Presence Teleoper Virtual Environ 6:603-616

Sparks GG, Sparks CW (2000) Violence, mayhem, and horror. Media Entertain Psychol Appeal 4:73-92
Tamborini R, Eastin MS, Skalski P, Lachlan K (2004) Violent virtual video games and hostile thoughts. J Broadcast Electron Media 48:335

Tannenbaum PH, Zillmann D (1975) Emotional arousal in the facilitation of aggression through communication. In: Berkowitz L (ed) Advances in experimental social psychology, vol 8. Academic Press, New York, pp 149-192

Thompson HS (1971) Fear and Loathing in Las Vegas: a savage journey to the heart of the American Dream. Random House, New York

Ventura M, Shute V, Zhao W (2013) The relationship between video game use and a performance-based measure of persistence. Comput Educ 60:52-58

Vorderer P, Hartmann T, Klimmt C (2003) Explaining the enjoyment of playing video games: the role of competition. In: Proceedings of the second international conference on entertainment computing, pp 1-9

Wang X, Ding X, Su S, Li Z, Riese H, Thayer JF et al (2009) Genetic influences on heart rate variability at rest and during stress. Psychophysiology 46:458-465

Wirth W, Hartmann T, Böcking S, Vorderer P, Klimmt C, Schramm H et al (2007) A process model of the formation of spatial presence experiences. Media Psychol 9(3):493-525

Witmer BG, Singer MJ (1998) Measuring presence in virtual environments: a presence questionnaire. Presence Teleoper Virtual Environ 7:225-240

Zillmann D, Weaver JB (1996) Gender-socialization theory of reactions to horror. In: Weaver JB, Tamborini R (eds) Horror films: current research on audience preferences and reactions. Routledge, $\mathrm{pp}$ $81-101$

Publisher's Note Springer Nature remains neutral with regard to jurisdictional claims in published maps and institutional affiliations. 